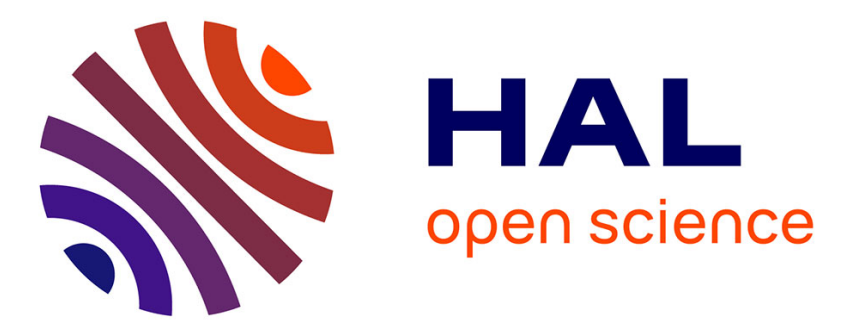

\title{
Résultats du projet Extraflo sur la comparaison des méthodes d'estimation des pluies extrêmes en France
}

L. Neppel, P. Arnaud, F. Borchi, Julie Carreau, F. Garavaglia, M. Lang, E. Paquet, Benjamin Renard, J.M. Soubeyroux, J.M. Veysseire

\section{- To cite this version:}

L. Neppel, P. Arnaud, F. Borchi, Julie Carreau, F. Garavaglia, et al.. Résultats du projet Extraflo sur la comparaison des méthodes d'estimation des pluies extrêmes en France. La Houille Blanche Revue internationale de l'eau, 2014, 2, p. 14 - p. 19. 10.1051/lhb/2014011 . hal-01062748

\section{HAL Id: hal-01062748 \\ https://hal.science/hal-01062748}

Submitted on 10 Sep 2014

HAL is a multi-disciplinary open access archive for the deposit and dissemination of scientific research documents, whether they are published or not. The documents may come from teaching and research institutions in France or abroad, or from public or private research centers.
L'archive ouverte pluridisciplinaire HAL, est destinée au dépôt et à la diffusion de documents scientifiques de niveau recherche, publiés ou non, émanant des établissements d'enseignement et de recherche français ou étrangers, des laboratoires publics ou privés. 


\title{
Résultats du projet Extraflo sur la comparaison des méthodes d'estimation des pluies extrêmes en France
}

\author{
Luc NEPPEL ${ }^{1}$, Patrick ARNAUD ${ }^{5}$, François BORCHI, Julie CARREAU ${ }^{1}$, Federico GARAVAGLIA ${ }^{4}$, \\ Michel LANG ${ }^{2}$, Emmanuel PAQUET ${ }^{4}$, Benjamin RENARD ${ }^{2}$, \\ Jean-Michel SOUBEYROUX ${ }^{3}$, Jean-Michel VEYSSEIRE ${ }^{3}$
}

\author{
1. HydroSciences Montpellier, cc MSEM, place Eugène Bataillon, 34095 Montpellier Cedex 5, neppel@msem.univ-montp2.fr \\ 2. IRSTEA, 5 rue de la Doua, CS 70077, 69626 Villeurbanne Cedex \\ 3. Météo-France, direction de la climatologie, 42 avenue Gaspard Coriolis, 31057 Toulouse Cedex 1 \\ 4. EDF-DTG, 21 avenue de l'Europe, BP 41 Cedex 9, 38040 Grenoble \\ 5. IRSTEA, 3275 Route de Cézanne, CS 40061, 13182 Aix en Provence Cedex 5
}

\begin{abstract}
RÉSUMÉ. - Cette communication présente une comparaison de trois types de méthodes de prédétermination des pluies extrêmes : 1) l'approche purement locale qui consiste à ajuster une distribution (lois généralisée des valeurs extrêmes GEV, de Pareto généralisée GP, ou par type de temps MEWP) sur un échantillon de pluies journalières relevées à un site cible, 2) les approches locales régionales qui consistent à enrichir l'échantillon d'observations du site cible par des données observées dans une région homogène et 3) les approches purement régionales que l'on peut utiliser en site non jaugé. Pour les deux dernières la distribution des pluies se déduit soit d'une analyse fréquentielle (AF) basée sur la théorie des valeurs extrêmes, soit de simulations du modèle SHYREG de génération stochastique de pluie. La comparaison s'appuie sur un jeu de 1568 stations pluviométriques issues de Météo-France et d'EDF dans la moitié Sud de la France. Le protocole de comparaison a été défini à partir des différents échantillons de calage/validation. Les critères de comparaison permettent de juger du biais et de la variabilité des quantiles et intervalles de confiance.

Les principaux résultats soulignent :

1) localement, l'ajustement d'une loi GP sur les dépassements de seuil présente de meilleures performances qu'une loi GEV sur les maxima annuel.

2) la loi exponentielle est inadaptée en particulier en région méditerranéenne.

3) de faibles performances des approches locales comparées aux approches locales-régionales et purement régionales, exceptée pour l'AF par type de temps MEWP comparable à l'approche locale-régionale hors région méditerranéenne avec plus de 10 ans pour le calage.

4) des performances comparables pour les approches locales régionales des méthodes d'AF et celle basée sur les simulations du modèle stochastique de pluie SHYREG.

5) une dégradation assez faible des critères lorsque l'on passe d'un ajustement de loi en version locale-régionale à purement régionale.
\end{abstract}

Mots-clés : pluies extrêmes, analyse fréquentielle, comparaison de méthodes, approche régionale.

\section{Comparison of extreme rainfall frequency analysis methods in France}

\begin{abstract}
This paper focuses on a comparison of 3 families of extreme rainfall frequency analysis methods: 1) purely local approaches which consist in fitting a probability distribution function (pdf) to a sample of observed daily rainfall, 2) local-regional approaches where the sample of annual maxima daily rainfall at the target site is enlarged by rainfall observations in a surrounding homogeneous neighbourhood and 3) purely regional approaches for estimating the extreme rainfall pdf at an ungaged site. For 2) and 3) the extreme rainfall pdf can be estimated either by frequency analysis based on the extreme value theory or from the simulations of the French stochastical rainfall model named SHYREG. The comparison relies on a dataset of 1568 daily rainfall gages belonging to the French Weather Service (Météo-France) or the French Electricity Company (EDF) in the southern half of France. A comparison methodology and several comparison criteria have been defined to evaluate the bias and variances of the quantiles and the confidence intervals. The main results are: 1) At the local scale, the General Pareto Distribution fitted on a peak over threshold sample performs better that the Generalized Extreme Value pdf fitted on max annual sample, 2) the exponential pdf is clearly unsuitable in Mediterranean area, 3 the local approaches perform poorly compared to the local-regional and purely regional frequency analyses except for the MEWP weather pattern frequency analysis which is comparable to the local regional frequency analysis for non-Mediterranean climate and when the calibration sample size exceeds 10 years, 4) the local regional frequency analysis and the SHYREG method performances are comparable, and 5) there is only a weak decrease in performances when using a purely regional frequency analysis instead of a local-regional frequency analysis.
\end{abstract}

Key-words: extreme rainfall, frequency analysis, methods comparison, regional approach 


\section{INTRODUCTION}

Les méthodes de prédétermination des pluies extrêmes visent à associer un cumul ou une intensité de pluie à une période de retour ou une probabilité annuelle de nondépassement fixée. Ces méthodes sont nombreuses en France et les études comparatives permettant de préciser leurs avantages et inconvénients ne concernent que quelques-unes d'entre elles. On peut classer ces méthodes en deux catégories, suivant l'estimation de la fonction de répartition (fdr) des précipitations extrêmes. La première s'appuie sur la théorie des valeurs extrêmes (Coles, 2001, Beirlant et al., 2004) et offre un cadre paramétrique pour l'estimation de la fdr des maxima annuels de pluie ou des précipitations dépassant un seuil fixé. Dans la seconde on déduit la fdr des précipitations extrêmes à partir de modèles stochastiques de pluie. Ces modèles visent à reproduire le signal temporel de pluie à des pas de temps horaires ou journaliers. Ils sont utilisés pour générer de longues chroniques de pluies avec lesquelles on construit empiriquement la fdr des extrêmes pour différentes durées de cumul.

Ces deux familles de méthodes peuvent être appliquées au site cible où l'on souhaite estimer la fdr des pluies extrêmes suivant trois approches différentes : i) en version locale : les modèles probabilistes ou stochastiques sont calés en exploitant uniquement les observations du site cible, ii) en version locale-régionale : en plus des observations au site cible, on consolide l'échantillon avec les pluviomètres voisins appartenant à une zone supposée homogène, ou iii) en version purement régionale : le site cible n'est pas instrumenté et l'on estime la fdr des extrêmes uniquement à partir des observations des sites avoisinants.

Dans ce papier, on présente les résultats du projet ANR ExtraFlo portant sur une comparaison de méthodes s'appuyant soit sur la théorie des valeurs extrêmes soit sur un modèle stochastique de pluie, chaque méthode se déclinant en version locale, locale-régionale ou purement régionale. Dans la section suivante on présente les modèles candidats. Une troisième section est consacrée au cadre de la comparaison, aux critères de performances et aux données pluviométriques utilisées. Enfin sont présentés les résultats et discussions.

\section{MODÈLES DE PRÉDÉTERMINATION DES PLUIES EXTRÊMES}

\section{II.1. Modèles locaux d'analyse fréquentielle}

\section{II.1.1. Lois GEV et GP}

La théorie des valeurs extrêmes indique que la distribution asymptotique du minimum ou du maximum d'un très grand nombre de variables aléatoires indépendantes, identiquement distribuées est une loi GEV ou GP si le type d'échantillonnage sélectionne les valeurs supérieures à un seuil (Coles, 2001). Pour la loi GEV trois paramètres sont estimés (équation 1): le paramètre de position $\mu$, le paramètre d'échelle $\sigma$ et le paramètre de forme $\xi$.

$$
F(x, \mu, \sigma, \xi)=\exp \left\{-\left[1+\xi\left(\frac{x-\mu}{\sigma}\right)\right]^{-1 / \xi}\right\}
$$

Pour la loi GP, le seuil $\mu$ est fixé et deux paramètres sont estimés : le paramètre d'échelle $\sigma$ et le paramètre de forme $\xi$.

$$
F(x, \mu, \sigma, \xi)=1-\left(1+\frac{\xi(x-\mu)}{\sigma}\right)^{-1 / \xi}
$$

Où (1) et (2) ne sont définis que si $1+\frac{\xi(x-\mu)}{\sigma}>0$

Dans les deux cas, le paramètre de forme est lié au comportement de la queue de la distribution et définit trois sous-familles : Gumbel et Exponentielle si $\xi$ est nul pour respectivement (1) et (2), celle de Fréchet si $\xi$ est positif et celle de Weibull si $\xi$ est négatif. L'estimation des paramètres peut se faire suivant la méthode du maximum de vraisemblance, la méthode des moments, des moments pondérés ou des L-moments.

\section{II.1.2. Analyse saisonnière et par type de temps : MEWP}

La méthode MEWP (Multi Exponential Weather Pattern) a été introduite par Garavaglia et al. (2010). Elle s'appuie sur une analyse par type de temps et par saison pour homogénéiser les échantillons. La fdr des pluies extrêmes est une combinaison de distributions exponentielles calées selon huit types de temps sur la France et deux saisons. Les paramètres de chaque loi exponentielle sont obtenus selon la méthode du maximum de vraisemblance en utilisant les valeurs supérieures au quantile $70 \%$ des pluies journalières en chaque station.

\section{II.2. Modèles régionaux d'analyse fréquentielle}

\section{II.2.1. Loi régionale des pluies journalières maximales annuelles : LR}

Cette méthode fait l'hypothèse que les pluies maximales annuelles d'une même zone homogène sont distribuées suivant des lois GEV de même forme. On considère de plus ici que le voisinage est circulaire autour du site cible. Le rayon est optimisé pour respecter l'hypothèse d'homogénéité et inclure un maximum de stations. L'échantillon des pluies journalières max annuelles est constitué à chaque station. Après normalisation par la moyenne des pluies maximales annuelles, appelée index value, on constitue un échantillon régional indépendant. La loi régionale est une GEV calée sur cet échantillon par la méthode des moments pondérés. L'application au site cible suppose que l'on dénorme les quantiles obtenus par la loi régionale en multipliant par l'index value du site cible. Dans la version locale-régionale celui-ci est calculé à partir des observations du site cible, dans la version purement régionale il est interpolé spatialement à partir d'une régression multiple utilisant des covariables géographiques (Carreau et al., soumis).

\section{II.2.2. Interpolation spatiale des paramètres d'une GEV locale : SIGEV}

C'est une méthode purement régionale qui permet d'estimer en un site non jaugé la fdr des pluies maximales annuelles. Elle consiste à interpoler au site cible les paramètres d'une GEV calée par la méthode des moments pondérés sur les différents pluviomètres dont on dispose. La méthode d'interpolation est identique à celle de l'index value : pour chaque paramètre on construit une régression multiple utilisant des covariables géographiques.

\section{II.3. Modèle stochastique de génération d'épisodes pluvieux}

On utilise dans cette étude le modèle SHYPRE (Simulated HYdrographs for flood PRobability Estimation) développé 
depuis une vingtaine d'années à l'Irstea d'Aix en Provence (Cernesson et al. 1996, Arnaud et Lavabre 1999, Arnaud et al., 2007). L'objectif de SHYPRE est d'étudier les distributions de variables hydrologiques (pluies et débits) à partir d'une combinaison entre un modèle stochastique pour la pluie horaire et un modèle pluie-débit. Les fdr des pluies extrêmes sont empiriques, associées aux caractéristiques d'événements générés au pas de temps horaire sur de longues périodes de simulation. Ces fdr ne proviennent pas d'un ajustement de loi statistique sur les observations des pluies maximales. Les simulations effectuées correspondent à la simulation d'une centaine d'échantillons de 500 ans. La distribution empirique moyenne des cent distributions déduites des 500 ans de simulation permet d'obtenir une distribution centrale relativement peu soumise à l'échantillonnage des simulations. Le modèle peut être calé à partir d'observations horaires (modèle SHYPRE) ou journalières (modèle SHYREG). Dans cette étude est utilisée la version de 2009 (Cantet, 2009) avec un calage adapté sur des données journalières (SHYREG), en version localerégionale c.a.d en calant le générateur à l'aide des chroniques journalières du site cible ou en version purement régionale en déduisant les paramètres du modèle des caractéristiques des pluies journalières interpolées en tout point du territoire français (Sol et Desouches, 2005, Arnaud et al., 2008).

\section{DONNEES PLUVIOMETRIQUES ET CRITÈRES DE PERFORMANCES DES MODÈLES}

On dispose d'un échantillon de 1568 stations pluviométriques issues des bases des données de Météo-France (MF) et d'Electricité de France (EDF). Ces stations sont localisées dans la moitié Sud de la France, de la côte Atlantique jusqu'au Alpes en passant par les côtes Méditerranéennes. Seules les années avec moins de $10 \%$ de valeurs manquantes ont été retenues pour l'analyse fréquentielle, et pour SHYPRE on a écarté tous les mois avec plus de 10 jours manquants. La longueur des chroniques journalières retenues s'échelonne de 2 à 58 ans.

Le calage et la validation des modèles sont réalisés sur des jeux de données différents. Par validation on entend le calcul de critères de performances permettant de juger la justesse, ainsi que la stabilité des quantiles et des intervalles de confiance à l'échantillonnage. Ces critères font l'objet d'une description détaillée dans Renard et al. (2013). On les présente ici succinctement ainsi que la constitution des échantillons de calage et de validation qui leur sont associés.

\section{III.1. Critères de justesse}

Le premier indice de justesse, noté $F F$, consiste simplement à calculer la probabilité de non-dépassement de la valeur maximale observée $d_{\max }^{(i)}$ [England et al., 2003 ; Garavaglia et al., 2011]:

$$
F F^{(i)}=\hat{F}_{M}^{(i)}\left(d_{\max }^{(i)}\right)
$$

Si l'estimation est juste, la fréquence $\hat{F}_{M}^{(i)}$ estimée par le modèle pour $d_{\max }^{(i)}$ doit être égale à la «vraie» fréquence, notée $F^{(i)}$. On peut montrer que sous cette hypothèse de justesse, en chaque site $i$, la valeur $F F^{(i)}$ est une réalisation d'une distribution de Kumaraswamy (Kumaraswamy, 1980) de paramètres $\left(n^{(i)} ; 1\right): F F^{(i)} \sim K\left[n^{(i)} ; 1\right]$. Cette distribution a pour fonction de répartition (avec $n^{(i)}$ égal au nombre de données au site de validation $i$ ):

$$
F_{K}(t)=t^{n^{(i)}}, 0 \leq t \leq 1
$$

Le principe consiste à vérifier que les FF calculés sur les stations de validations suivent la loi théorique (4). Le critère $F F$ ne renseigne que sur la justesse des méthodes dans les extrêmes.

Le second indice de justesse s'intéresse aux quantiles de période de retour $T$ donnée. Pour cela, on définit l'indice $N_{T}$ qui consiste simplement à dénombrer à une station $i$ le nombre d'observations dépassant le quantile estimé [Gunasekara and Cunnane, 1992 ; Garavaglia et al., 2010]. Si l'estimation est juste, on peut montrer que la valeur $N_{T}^{(i)}$ est une réalisation d'une loi Binomiale avec probabilité de succès $1 / T$ et nombre d'essais $n^{(i)}$ (avec $n^{(i)}$ égal au nombre de données au site de validation $i): N_{T}^{(i)} \sim \operatorname{Bin}\left(n^{(i)}, 1 / T\right)$.

Ces deux critères sont calculés pour chaque station et l'on vérifie par un graphique probabilité-probabilité (pp-plot) que la distribution empirique du critère est proche de la distribution théorique attendue. Plus la courbe est proche de la $1^{\text {ière }}$ bissectrice plus le modèle est juste. L'information portée par ces pp-plot peut se résumer au travers d'un score compris entre 0 (modèle peu juste) et 1 (justesse parfaite) basé sur l'aire entre la courbe et la bissectrice.

Afin de juger du poids des informations locales et régionales dans les modèles purement régionaux et locauxrégionaux, on constitue aléatoirement un échantillon de calage et de validation comme il suit :

- $50 \%$ des stations sont utilisées pour le calage de l'information régionale, on utilisant toute la chronique disponible à ces stations

- pour chacune des stations restantes, on choisit aléatoirement $n$ années utilisées pour le calage de l'information locale : $n$ varie de 5 à 20 années de façon à évaluer le poids de l'information locale par rapport à l'information régionale. - la validation des méthodes est effectuée sur les stations non utilisées pour le calage de l'information régionale et sur les années restantes non utilisées pour le calage de l'information locale.

\section{III.2. Critères de stabilité}

La stabilité des quantiles est décrite avec un indice noté $\mathrm{SPAN}_{\mathrm{T}}$ décrivant la différence relative entre les quantiles de période de retour $\mathrm{T}$ estimés par un modèle donné sur deux jeux de calage distincts $\mathrm{C} 1$ et $\mathrm{C} 2$. Les quantiles sont d'autant plus stables que le $\mathrm{SPAN}_{\mathrm{T}}$ est proche de zéro.. On calcule un critère par station et la moyenne normée des $\operatorname{SPAN}_{\mathrm{T}}$ de toutes les stations. Cette dernière représente un score qui résume la stabilité du quantile $\mathrm{T}$ sur toutes les stations : il vaut 0 si l'estimation est parfaitement stable et 1 si l'estimation est très instable.

La stabilité des intervalles de confiances d'un quantile fixé est mesurée par le taux de recouvrement de deux intervalles de confiances calculés avec deux périodes de calage différentes. Cet indice noté $\mathrm{COVER}_{\mathrm{T}}$ varie entre 0 et 1 correspondant respectivement à aucun recouvrement et à un recouvrement parfait (Garavaglia et al., 2011).

Pour les modèles locaux-régionaux et purement régionaux, la stabilité des quantiles et des intervalles de confiance peut être jugée par rapport à l'information locale utilisée dans les modèles et par rapport à l'information régionale. Le tableau 1 détaille la construction des jeux $\mathrm{C} 1$ et $\mathrm{C} 2$ pour chaque cas. 
Tableau 1 : Construction des échantillons pour le calcul des critères de stabilité des quantiles.

\begin{tabular}{|c|c|}
\hline Stabilité à l'information régionale & Stabilité à l'information locale \\
\hline $\begin{array}{l}\text { - On utilise } 33 \% \text { des stations pour constituer un premier jeu } \\
\text { de calage de l'information régionale (R1) et } 33 \% \text { d'autres } \\
\text { stations pour le second jeu (R2). } \\
\text { - Sur les } 33 \% \text { de stations restantes, on tire } n \text { années } \\
\text { au hasard pour caler l'information locale. } \\
\text { - On construit deux jeux de calage } \mathrm{C} 1=\mathrm{R} 1+\mathrm{L} \text {, } \\
\text { et } \mathrm{C} 2=\mathrm{R} 2+\mathrm{L} \text {. Ils diffèrent par l'information régionale, } \\
\text { mais utilisent la même information locale. }\end{array}$ & $\begin{array}{l}\text { - L'information régionale est calée sur } 33 \% \text { des stations (R) } \\
\text { - On tire } n \text { années au hasard dans les } 66 \% \text { de stations } \\
\text { restantes pour le premier jeu de calage de l'information } \\
\text { locale (L1), et } n \text { autres années pour le second jeu (L2). } \\
\text { - On construit deux jeux de calage } \mathrm{C} 1=\mathrm{R}+\mathrm{L} 1 \text {, } \\
\text { et } \mathrm{C} 2=\mathrm{R}+\mathrm{L} 2 . \text { Ils diffèrent par l'information locale } \\
\text { mais utilisent la même information régionale. }\end{array}$ \\
\hline
\end{tabular}

\section{RESULTATS}

On présente d'abord les performances concernant la justesse puis la stabilité des quantiles et intervalles de confiance. Pour chaque critère on considère d'abord les méthodes d'AF purement locales, puis on retient les meilleures méthodes que l'on compare ensuite aux approches ayant une composante régionale.

\section{IV.1. Analyse de la justesse}

\section{IV.1.1. Méthodes d'AF purement locales}

Une première comparaison porte sur les méthodes GP et GEV qui diffèrent par la méthode d'échantillonnage. Le critère $N_{10}$ ne montre pas de différence réelle entre ces méthodes, le critère $N_{100}$ donne quant à lui une légère préférence à la loi GP. La méthode GP donne de meilleurs résultats pour le score $F F$, surtout avec de petits échantillons.; quand l'échantillon de calage décroît, la méthode GEV perd davantage de précision pour l'estimation des valeurs extrêmes que la méthode GP. Donc le modèle GP donne des résultats plus fiables pour les grandes durées de retour que le modèle GEV, surtout pour de petits échantillons, et c'est lui que l'on garde pour la suite des comparaisons.

Les scores caractérisant la fiabilité $\left(\mathrm{FF}\right.$ et $\mathrm{N}_{10}$ ) sont alors nettement meilleurs pour les trois méthodes SHYREG, MEWP et GP que pour la loi EXPO et assez proches entre eux (voir Tableau 2).

Globalement, SHYREG obtient des résultats légèrement meilleurs à MEWP et GP . La loi EXPO manquant manifestement de justesse n'est pas adaptée. On observe également une sous estimation par MEWP, particulièrement visible sur le $\mathrm{N}_{10}$.

\section{IV.1.2. Méthodes purement locales \& avec une approche régionale}

Une première analyse des résultats (Figure 1) montre que les modèles les plus sensibles au nombre d'années de calage

Tableau 2 : Comparaison des critères $N_{10}$ et FF entre quatre méthodes (pour les séries de plus de 50 ans).

\begin{tabular}{|l|c|c|}
\hline \multirow{2}{*}{} & \multicolumn{2}{|c|}{ Calage (25 années) - Validation (25 années) } \\
\cline { 2 - 3 } & $\mathbf{F F}$ & $\mathbf{N}_{\mathbf{1 0}}$ \\
\hline SHYREG & 0,95 & 0,95 \\
\hline MEWP & 0,92 & 0,85 \\
\hline GP & 0,93 & 0,91 \\
\hline GEV & 0,88 & 0.91 \\
\hline EXPO & 0,69 & 0,76 \\
\hline
\end{tabular}

sont d'abord les méthodes d'AF purement locales, ici GP suivi de MEWP. La loi régionale en version locale-régionale et SHYREG sont les moins sensibles par rapport aux précédents, qu'il s'agisse de la justesse du corps des distributions $\left(\mathrm{N}_{10}\right)$ ou des extrêmes $\left(\mathrm{N}_{100}, \mathrm{FF}\right)$.

On remarque ensuite qu'avec plus de 10 ans de données de calage, les performances du modèle MEWP se rapprochent des méthodes avec une composante régionale, seule la GP montre une justesse plus faible.

On note également une meilleure performance de la loi régionale par rapport à la loi locale-régionale pour estimer les quantiles de période de retour supérieure aux années disponibles pour le calage. L'approche locale-régionale semble plus juste que l'approche régionale, pour estimer les extrêmes.

Entre les deux approches purement régionales, la loi régionale se démarque nettement de la méthode SIGEV, qui figure parmi les méthodes les moins justes.

\section{IV.2. Stabilité des quantiles et intervalles de confiances}

L'analyse de la stabilité est réalisée d'abord entre méthodes purement locales, puis entre méthodes ayant toutes une composante locale et enfin entre méthodes ayant toutes une composante régionale.

\section{IV.2.1. Méthodes d'AF purement locales}

Le critère $S P A N_{10}$ ne montre pas une réelle différence de stabilité entre les méthodes GEV et GP, mais les quantiles de durée de retour 100 ans estimés avec l'estimateur GP sont plus stables que ceux estimés avec l'estimateur GEV. Cette différence de stabilité pour la durée de retour 100 ans est plus visible pour les stations ayant des estimations de quantiles élevées ou basses. De plus, le critère $C O V E R_{T}$ montre que l'estimation de la variance est bien meilleure avec la méthode GP qu'avec la méthode GEV pour les deux durées de retour 10 et 100 ans. Comme pour la justesse, on retient donc la méthode GP pour la suite des comparaisons.

Étant donné d'autre part que la loi Expo s'était distinguée par sa moindre justesse, seuls les résultats des critères SPAN $_{10}$ et SPAN $_{100}$ pour les lois SHYREG, MEWP et GP sont présentés (voir Tableau 3). Les meilleurs résultats sont obtenus pour les lois MEWP et SHYREG, avec un léger avantage pour celle-ci, les écarts s'accentuant pour le quantile 100 ans par rapport au quantile 10 ans. La méthode GP montre quant à elle une forte sensibilité de ses résultats à la taille de l'échantillon.

\section{IV.2.2. Méthodes ayant une composante locale}

Les modèles candidats sont la loi régionale en version locale-régionale, MEWP, GP et SHYREG. La stabilité 


\begin{tabular}{|c|c|c|c|c|c|}
\hline - Pure Reg & Reliability & & $N_{10}$ & $N_{100}$ & FF \\
\hline L Loc Reg 5 & & Pure Reg & 0.8384 & 0.9529 & 0.8838 \\
\hline - Loc Reg 10 & $\mathrm{~N}_{100}$ & Loc Reg 5 & 0.7817 & 0.9229 & 0.9200 \\
\hline _ Loc Rea 20 & & Loc Reg 10 & 0.8461 & 0.9197 & 0.9170 \\
\hline LOc Keg 20 & & Loc Reg 20 & 0.8893 & 0.9441 & 0.9609 \\
\hline SIGEV & & SIGEV & 0.7207 & 0.6536 & 0.8077 \\
\hline MEWP 10 & & MEWP 5 & 0.7380 & 0.9273 & 0.8583 \\
\hline MEWP 10 & & MEWP 10 & 0.8328 & 0.9330 & 0.9014 \\
\hline MEWP 20 & & MEWP 20 & 0.8774 & 0.9061 & 0.9566 \\
\hline GP 5 & & GP 5 & 0.6915 & 0.8295 & 0.7677 \\
\hline GP 10 & . & GP 10 & 0.7530 & 0.8563 & 0.8115 \\
\hline GP 20 & & GP 20 & 0.8431 & 0.8883 & 0.9028 \\
\hline SHYREG 5 & $\mathbf{F F}$ & SHYREG 5 & 0.7813 & 0.9325 & 0.8730 \\
\hline SHYREG 10 & & SHYREG 10 & 0.8567 & 0.9517 & 0.8928 \\
\hline SHYREG 20 & & SHYREG 20 & 0.9060 & 0.9688 & 0.9387 \\
\hline
\end{tabular}

Figure 1 : Synthèse des critères de justesse pour la loi régionale en version purement régionale (Pure Reg), en version locale-régionale (Loc Reg), pour la méthode d'interpolation (SIGEV), pour les méthodes locales d'AF (MEWP et GP) et enfin pour le générateur stochastique en version local régionale (SHYREG). Les chiffres suivants les méthodes indiquent le nombre d'années utilisées pour le calage de l'information locale.

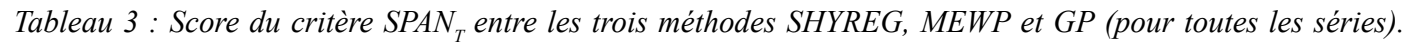

\begin{tabular}{|l|c|c|c|c|c|c|}
\hline \multirow{2}{*}{ SPAN $_{\mathbf{T}}$} & \multicolumn{2}{|c|}{ Calage - Validation (10 années) } & \multicolumn{2}{c|}{ Calage - Validation (15 années) } & \multicolumn{2}{c|}{ Calage - Validation (25 années) } \\
\cline { 2 - 7 } & $\mathbf{T}=\mathbf{1 0}$ ans & $\mathbf{T}=\mathbf{1 0 0}$ ans & $\mathbf{T}=\mathbf{1 0}$ ans & $\mathbf{T}=\mathbf{1 0 0}$ ans & $\mathbf{T}=\mathbf{1 0}$ ans & $\mathbf{T}=\mathbf{1 0 0}$ ans \\
\hline GP & 0,82 & 0,65 & 0,86 & 0,70 & 0,88 & 0,76 \\
\hline MEWP & 0,86 & 0,81 & 0,89 & 0,85 & 0,92 & 0,89 \\
\hline SHYREG & 0,87 & 0,84 & 0,90 & 0,87 & 0,93 & 0,91 \\
\hline
\end{tabular}

des quantiles par rapport à l'information locale montre (Figure 2) :

- La loi régionale et SHYREG sont les plus stables, SHYREG ayant les meilleures performances pour les quantiles élevés

- A partir de 20 ans de données de calage, le modèle MEWP a une stabilité équivalente à la loi régionale et SHYREG, pour les quantiles décennaux et centennaux

- Si l'on augmente le nombre d'années de calage, la stabilité augmente, la sensibilité à l'effectif de calage étant plus marquée pour la loi GP et pour les quantiles élevés

On note que la stabilité à l'information locale de la loi régionale en version locale-régionale est indépendante de la fréquence. En effet on ne juge ici que de la sensibilité des quantiles et intervalles de confiance à l'index value, son influence est la même pour toutes les périodes de retour.

\section{IV.2.3. Méthodes ayant une composante régionale}

Les modèles candidats sont la loi régionale en version locale-régionale ou purement régionale et la méthode SIGEV d'interpolation des paramètres d'une GEV locale. On constate (Figure 2) une légère diminution de la stabilité des quantiles et des intervalles de confiance quand on applique la loi régionale en version purement régionale par rapport à la version locale-régionale. Cependant ces deux modèles montrent une stabilité bien meilleure que la méthode SIGEV qui est beaucoup plus sensible à l'échantillon des stations disponibles pour l'interpolation.

\section{CONCLUSIONS}

Cet article synthétise les résultats d'une comparaison des méthodes de prédétermination des pluies extrêmes basées sur l'AF et la théorie des valeurs extrêmes d'une part et l'utilisation d'un générateur stochastique d'autre part. Des critères spécifiques pour juger des performances de justesse et de stabilité des méthodes ont été proposés, ainsi qu'un protocole permettant de prendre en compte la densité d'information disponible pour le calage des différentes méthodes. Les recommandations que l'on peut en tirer sont les suivantes.

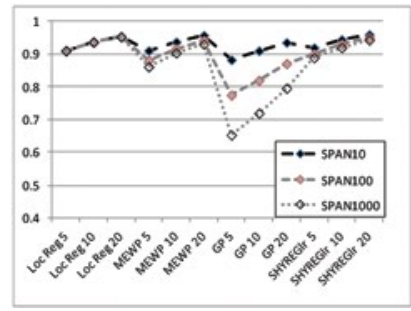

(a)

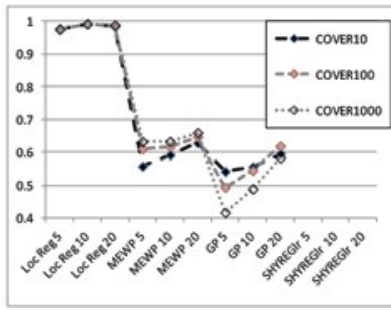

(b)

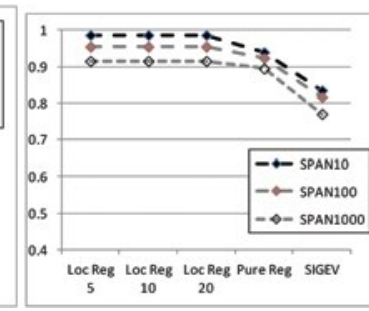

(c)

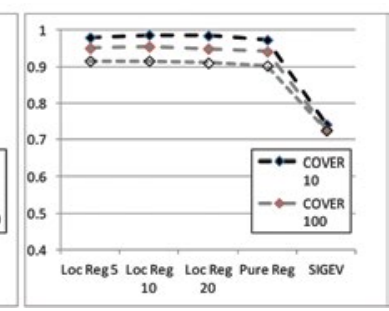

(d)

Figure 2 : Synthèse des critères de stabilité à l'information locale ( $a: S P A N, b: C O V E R)$ et à l'information régionale (c : SPAN, $d:$ COVER). 
Pour les périodes de retour inférieures à 10 ans, les performances des différentes méthodes sont assez proches dès qu'on dispose au moins de 10 ans de données pour le calage des méthodes d'AF.

Pour une période de retour de 100 ans, la loi régionale, la méthode d'AF par type de temps MEWP avec plus de 10 ans de données de calage et le générateur stochastique SHYREG donnent les meilleurs résultats tant au niveau de la justesse que de la stabilité des quantiles. On notera une stabilité plus grande de SHYREG pour les quantiles $\mathrm{T}=1000$ ans, mais cette méthode n'a pas été jugée sur ces intervalles de confiance contrairement aux autres. Les méthodes d'ajustement local de lois GEV, GP ou exponentielle sont à éviter quand on s'intéresse aux quantiles centennaux, sauf à adopter une approche locale-régionale. En région méditerranéenne, la loi exponentielle est à proscrire et l'approche MEWP est moins performante.

Pour l'estimation de quantiles en site non jaugé, les méthodes régionales reposant sur l'estimation d'une loi régionale sont à préférer aux méthodes basées sur l'interpolation spatiale des paramètres d'une GEV qui sont moins justes et moins stables, pour tous les niveaux de fréquences.

Enfin ces conclusions sont limitées au cadre de cette étude. Elles ne concernent que les précipitations locales et non pas les pluies de bassin ou de lames d'eau, ni des zones géographiques avec une densité de poste très réduite comme en haute-montagne. Les résultats ont été obtenus à partir de l'analyse de pluie journalière. La plupart des méthodes peuvent s'adapter aux précipitations infra-journaliers mais la hiérarchie des méthodes risque d'être différente en fonction de leur sensibilité à la densité du réseau, qui est ici réduite. Seule la méthode SHYREG permet directement une estimation des quantiles de pluies pour des durées de $1 \mathrm{~h}$ à $72 \mathrm{~h}$ sur tout le territoire métropolitain.

\section{BIBLIOGRAPHIE}

ARnaud P., Fine J.A., LAVABre J. (2007) - An hourly rainfall generation model applicable to all types of climate. Atmospheric Research. 85 : 230-242

Arnaud P., Lavabre J. (1999) — Using a stochastic model for generating hourly hyetographs to study extreme rainfalls. Hydrol. Sci. J. 44 : 433-446
Arnaud P., Lavabre J., Sol B., Desouches C. (2008) Regionalization of an hourly rainfall generating model over metropolitan France for flood hazard estimation. Hydrol. Sci. J. $53: 34-47$

Beirlant J., Goegebeur Y., Teugels J. And Segers J. (2004) Statistics of extremes. Theory and applications. Wiley Series in Probability and Statistics. Wiley

CANTET P. (2009) - Impacts du changement climatique sur les pluies extrêmes par l'utilisation d'un générateur stochastique de pluies. Thèse de doctorat de l'Université de Montpellier 2

Carreau J., Neppel L., Arnaud P., Cantet P. (2013) - Extreme Rainfall Analysis at Un-Gauged Sites in the South of France : Comparison of Three Approaches. Journal de la Société Française de Statistique, soumis

Cernesson F., Lavabre J., Masson J.M. (1996) - Stochastic model for generating hourly hyetographs. Atmospheric Research. 42 (1-4)

Coles S. (2001) - An Introduction to Statistical Modeling of Extreme Values. Springer Series in Statistics, ed. SpringerVerlag. London: Springer-Verlag. 210 p.

England J. F., R. D. Jarrett And J. D. Salas (2003) - Data-based comparisons of moments estimators using historical and paleoflood data. J. Hydrol. 278(1-4) : 172-196

Garavaglia F., J. Gailhard, E. Paquet, M. Lang, R. Garcon And P. BERNARDARA (2010) — Introducing a rainfall compound distribution model based on weather patterns sub-sampling. Hydrol. Earth Syst. Sci. 14(6) : 951-964

Garavaglia F., M. Lang, E. Paquet, J. Gailhard, R. Garcon And B. RENARD (2011) - Reliability and robustness of a rainfall compound distribution model based on weather pattern subsampling. Hydrology and Earth System Sciences. 15(2) : 519-532

Gunasekara T. A. G. And C. Cunnane (1992) - Split Sampling Technique for Selecting a Flood Frequency-Analysis Procedure. J. Hydrol. 130(1-4) : 189-200

KUMARASWAMY P. (1980) - A generalized probability density function for double-bounded random processes. J. Hydrol. 46(1-2) : 79-88

Renard B., K. Kochanek, M. Lang, F. Garavaglia, E. Paquet, L. Neppel, K. Najib, J. Carreau, P. Arnaud, Y. Aubert, F. Borchi, J. M. Soubeyroux, S. Jourdain, J. M. Veysseire, E. Sauquet, T. Cipriani And A. Auffray (2013) - Databased comparison of frequency analysis methods: a general framework. Water Resources Research, in press

Sol B. Et Desouches C. (2005) - Spatialisation à résolution kilométrique sur la France de paramètres liés aux précipitations. Technical Report 3, Météo-France 